\title{
Red Cell Autoantibodies in a Thalassemia Major Patient
}

\section{Nidhi B*}

Department of Transfusion Medicine, BJ Medical College, India

*Corresponding author: Nidhi Bhatnagar, Associate Professor, Department of Transfusion Medicine, BJ Medical College, Ahmedabad, India, Tel: 9825097675; Email: bhatnagarnidhi@ymail.com

\section{Case Report}

Volume 3 Issue 2

Received Date: September 12, 2019

Published Date: September 23, 2019

DOI: $10.23880 /$ hij-16000145

\section{Abstract}

$\beta$ Thalassemia is one of the most prevalent autosomal disorders, which affects more than $4,00,000$ new born per year worldwide. In India, the carrier rate of $\beta$ thalassemia varies from 3-17\%. The development of red blood cell antibodies (both allo-and auto) remains a major problem in thalassemia major patients. Red cell autoantibodies appear less frequently but they can result in clinical hemolysis called autoimmune hemolytic anemia (AIHA) and result in difficulty in cross-matching blood. Patients with autoantibodies may have a higher transfusion rate and often require immunosuppressive drugs or alternative treatments including intravenous immunoglobulin (IVIg) and rituximab (antiCD20 monoclonal antibody). We describe a case of 19 months old male patient, who was admitted with diagnosis of thalassemia major and had received multiple transfusions from several centers. He developed hemolytic anemia and cell panel was suggestive of autoimmune hemolytic anemia. Finding a compatible blood unit for him was a major challenge. To maintain his hemoglobin level, least incompatible blood unit was issued under steroid cover. He required blood transfusion every 3-4 weeks and each time least incompatible blood was issued to maintain hemoglobin level. Oral steroids including rituximab were given post transfusion for 1 week.

Keywords: Autoimmunization; Multitransfused; Thalassemia

Abbreviations: AIHA: Autoimmune Hemolytic Anemia; IVIG: Intravenous Immunoglobulin; MF: Mixed Field; DCT: Direct Coomb's Test; ICT: Indirect Coomb's Test.

\section{Introduction}

Thalassemia is a congenital hemolytic disorder, caused by a partial or complete defect in $\alpha$ or $\beta$ globin chain synthesis. Thalassemia is considered as the most common genetic disorder world-wide with a particularly high frequency in a broad belt, extending from the Mediterranean basin through the Middle East (Iran), India and Southeast Asia [1]. The carrier rate varies between 5.5 and $\geq 9 \%$. It was estimated that $1000 / 1.5$ million per year live births have $\beta$-thalassemia [2]. In India, the carrier rate of $\beta$-thalassemia varies from $3-17 \%$ [2]. The overall rate of autoimmunization in thalassemia patients has been reported to be $5-30 \%$ in the world, which is mostly contributed by the autoimmunization to minor blood group antigens [3]. Among Asians, the incidence of red cell autoimmunization is $22 \%$ [3]. In India, it is estimated that nearly 8000-10000 new thalassemics (homozygous) are born every year. The $\beta$ thalassemia gene is found more commonly in Punjabis, Sindhis, Bengalis, and Gujaratis. The recommended treatment for 
$\beta$-thalassemia major is regular blood transfusion every 3 to 4 weeks. The development of anti-red cell antibodies (alloantibodies and/or autoantibodies) can significantly complicate transfusion therapy. Currently, providing compatible blood for alloimmunized thalassemia patients presents a major challenge. Transfusion of extended phenotype matched blood can be useful in the prevention of alloimmunization [4]. Red cell autoantibodies appear less frequently, but they can result in clinical hemolysis called autoimmune hemolytic anemia (AIHA), and in difficulty in finding a compatible blood unit. The pathogenesis of erythrocyte autoantibody formation following transfusion is also not well understood, though, clinical evidence of autoimmune hemolytic anaemia has been seen with high amounts of RBC-associated IgG [5]. Patients with autoantibodies may have a higher transfusion rate and often require immunosuppressive drugs or alternative treatments including intravenous immunoglobulin (IVIg) and rituximab.

\section{Case Report}

A 19 months old male patient, known case of $\beta$ thalassemia major who was the recipient of regular transfusions since 11 months of age, came to our hospital for treatment. The laboratory workup showed positive Direct and Indirect Coomb's Test (DCT and ICT), hemoglobin of $5.3 \mathrm{~g} / \mathrm{dl}$, Total bilirubin level of $3.8 \mathrm{mg} / \mathrm{dl}$. As the patient became ICT-positive, finding a compatible donor blood unit was the major challenge. The patient was thoroughly investigated and all the necessary serological investigations were performed, which included major blood grouping (forward and reverse) and Rh phenotyping.

Apart from DCT \& ICT, Antibody screening, and identification were also done subsequently. All of these tests were performed by using LISS Coomb's ID Gel Cards from Diamed. Elution was done to identify the type of antibody coated over the red cells. Non-specific mixed field (mf) pan-agglutination reaction was seen on 11 cell antibody identification panel. Rh phenotype matched blood was given but it showed +1 reactivity in compatibility testing. Also, finding an antigen profilematched donor was a major issue.

He was started on Prednisolone $1 \mathrm{mg} / \mathrm{kg} /$ day for two weeks and then tapering dose. Thus, the combined approach of both drug therapy including rituximab, and regular transfusion of red cell antigen (major as well as minor)-matched blood, helped to solve the challenging case of thalassemia complicated with AIHA.
The treatment of thalassemia major is blood transfusions to maintain the hemoglobin level [6]. Although life expectancy of thalassemic patients has increased by the progress achieved in the lines of management of thalassemia, but still the majority of our patients are young, as $66.8 \%$ were younger than 12 years.

\section{Discussion}

In this report, we evaluated the importance of doing extended red cell antigen phenotyping for chronically transfused thalassemia patients. We also highlight the effectiveness of rituximab in thalassemia cases, complicated by AIHA. Rituximab is a chimeric monoclonal antibody against the protein $\mathrm{CD} 20$, which is found on the surface of B cells. Rituximab destroys B cells, and is, therefore, used to treat diseases, which are characterized by excessive numbers of B cells, overactive B cells, or dysfunctional B cells, which is seen in AIHA [6]. The factors for autoimmunization are complex, which involves the RBC antigenic difference between the blood donor and the recipient; the recipient's immune status; and the immunomodulatory effect of the allogeneic blood transfusions. The distribution of various blood groups antigens varies amongst individuals in any given population. Therefore, there is a variable degree of disparity amongst the donors and the recipient as far as minor blood group antigens are concerned, which are not tested for routine transfusions. As a result, autoimmunization can take place during the transfusion management. As regards the risk of autoimmunization, all patients receiving multiple transfusions should be typed for clinically important blood group antigens including ABO, Rh, Kell, Kidd and Duffy systems, etc [7]. To cause AIHA, transfusion-related immunomodulation plays an important role, in which the patients produce autoantibodies that bind to erythrocytes, leading to their destruction and a resultant anemia. There are very few studies that highlight the concept of AIHA in patients with thalassemia. A study of 319 thalassemic children, by Dhawan $\mathrm{H}$, et al. found the rate of developing autoantibodies to be $28.2 \%$ [1]. AIHA represents a failure of self-tolerance. Although many cases of AIHA are idiopathic, some of the conditions associated with AIHA are autoimmune disorders [8-10].

\section{Conclusion}

Complicated cases of thalassemia, which develop autoimmunization along with refractory autoimmunization leading to AIHA, are very difficult to 
treat. Corticosteroid drug therapy followed by IVIg and rituximab with the regular transfusion of extended red cell phenotype-matched blood is a very effective regimen for such patients. This combined approach could be a good regimen for other complicated cases of auto and/or alloimmunization also, who require regular periodic blood transfusions.

\section{References}

1. Dhawan HK, Kumawat V, Marwaha N, Sharma RR, Sachdev S, et al. (2014) Alloimmunization and autoimmunization in transfusion dependent thalassemia major patients: Study on 319 patients. Asian J Transfus Sci 8(2): 84-88.

2. Agarwal S, Gupta A, Gupta UR, Sarwai S, Phadke S, et al. (2003) Prenatal diagnosis in beta-thalassemia: An Indian experience. Fetal Diagn Ther 18(5): 328-332.

3. Singer ST, Wu V, Mignacca R (2000) Alloimmunization and erythrocyte autoimmunization in transfusion dependent thalassemia patients of predominantly Asian descent. Blood 96(10): 33693373.

4. Davari K, Soltanpour M (2016) Study of alloimmunization and autoimmunization in Iranian $\beta$ thalassemia major patients. Asian J Transfus Sci 10(1): 88-92.
5. Elhence P, Solanki A, Verma A(2014) Red Blood Cell Antibodies in Thalassemia Patients in Northern India: Risk Factors and Literature Review. Indian J Hematol Blood Transfus 30(4): 301-308.

6. Shanafelt TD, Madueme HL, Wold RC, Tefferi A (2003) Rituximab for Immune Cytopenia in Adults: Idiopathic Thrombocytopenic Purpura, Autoimmune Hemolytic Anemia, and Evans Syndrome. Mayo Clin Proc 78(11): 1340-1346.

7. Wonke B (2001) Clinical Management of betathalassemia Major. Semin Hematol 38(4): 350-359.

8. Wang LY, Liang DC, Liu HC, Chang FC, Wang CL, et al. (2006) Alloimmunization among patients with transfusion-dependent thalassemia in Taiwan. Transfus Med 16(3): 200-203.

9. Ameen R, Al Shemmari S, Al Humood S, Chowdhury RI, Al Eyaadi O, et al. (2003) RBC alloimmunization and autoimmunization among transfusion dependent Arab thalassemia patients. Transfusion 43(11): 16041610.

10. Noor Haslina MN, Ariffin N, Illuni Hayati I, Rosline $\mathrm{H}$ (2007) Red cell autoantibodies among thalassaemia patients in Hospital Universiti Sains Malaysia. Singapore Med J 48(10): 922-925. 